\title{
Effects of Nuchal Cord on Maternal \& Foetal Outcome
}

\author{
Neeru Verma ${ }^{1}$, Sangeeta Singh ${ }^{2}$, Jyoti Sakral ${ }^{3}$, Naresh Khajuria ${ }^{4}$, Naresh Sharma ${ }^{5}$ \\ ${ }^{1}$ Department of Health and Medical Education, Sub District Hospital, Akhnoor, J \& K, India. ${ }^{2}$ Department of Health and \\ Medical Education, Sub District Hospital, Akhnoor, J \& K, India. ${ }^{3}$ Department of Health and Medical Education, Sub \\ District Hospital, Akhnoor, J \& K, India. ${ }^{4}$ Department of Health and Medical Education, Sub District Hospital, Akhnoor, \\ J \& K, India. ${ }^{5}$ Department of Health and Medical Education, Sub District Hospital, Akhnoor, J \& K, India.
}

\section{ABSTRACT}

\section{BACKGROUND}

Umbilical cord is the connecting link between the foetus and the placenta through which foetal blood flows to and from the placenta. It extends from the foetal umbilicus to the foetal surface of the placenta. Excessively long cords are linked with cord entanglement. Nuchal cord occurs when umbilical cord gets wrapped around foetal neck $360^{\circ}$. Single loop around neck is reported in $20-34 \%$ cases. Cord compression is one of the postulated factors for birth asphyxia. It has become a cause of concern for rising primary $\mathrm{C}$ Section rate. We wanted to determine the actual threat nuchal cord poses to the mother as well as to the foetus and identify various factors which should be considered for safe delivery in these patients.

\section{METHODS}

This study was conducted in the Department of Health and Medical Education, SubDistrict Hospital, Akhnoor, J \& K. We included 100 cases in the test group in which nuchal cord was identified during delivery, delivered either vaginally or by $\mathrm{C}$ section. These cases were compared with 100 controls delivered without nuchal cord. We had to observe 462 patients for finding out 100 cases. Incidence of nuchal cord in our study is $21.64 \%$. Patients profile was noted on proforma and various variables were compared such as age, parity, gestational age, mode of delivery, duration of labour, length of cord, number of loops and prior USG detection of cord were noted in every case. Foetal outcome as observed in terms of gender, birth weight and Apgar score was recorded.

\section{RESULTS}

Most of the patients belonged to the age group 21-25 years with a mean age of 25.56 years. Incidence of nuchal cord is $21.64 \%$. As the number of turns increased, incidence fell progressively, but as the number of loops increased length of cord also increased. Only 36 loops were pre detected on USG. Maximum number of patients had normal vaginal delivery in both groups, but the rate of $\mathrm{C}$ section was higher in test group and difference was statistically significant $(0.028)$. When variables of newborn were compared, no significant difference was seen in sex and weight of the baby. Meconium stained liquor was more in nuchal cord group and although low Apgar score was more in nuchal cord group, the difference was not statistically significant.

\section{CONCLUSIONS}

Nuchal cord is a common feature observed during delivery and is seen almost in 20$25 \%$ cases. Ante natal diagnosis of nuchal cord is possible with USG and colour doppler imaging. Elective $\mathrm{C}$ section for nuchal cord is a wrong practice. Patients should be assessed holistically including age, parity, gestational age, Bishop score and signs of foetal distress. Vigilant ante partum and intra partum monitoring is required in every case. Nuchal cord is potential cause of perinatal distress and rarely significant long-term neurodevelopmental consequences are seen in the foetus.

\section{KEY WORDS}

Nuchal Cord Apgar Score Entanglement Pregnancy.
Corresponding Author:

Dr. Neeru Verma,

House No. 10, Lane No.1,

Suraksha Vihar,

Near BSF Campus Paloura Top,

Jammu, J \& K, India.

E-mail: neeru26verma@gmail.com

DOI: $10.14260 / \mathrm{jemds} / 2020 / 448$

How to Cite This Article:

Verma N, Singh S, Sakral J, et al. Effects of nuchal cord on maternal \& foetal outcome. J. Evolution Med. Dent. Sci. 2020;9(29): 2058-2062, DOI:

$10.14260 /$ jemds/2020/448

Submission 18-01-2020,

Peer Review 12-06-2020

Acceptance 19-06-2020,

Published 20-07-2020.

Copyright (C) 2020 JEMDS. This is an open access article distributed under Creative Commons Attribution License [Attribution 4.0 International (CC BY 4.0)] 


\section{BACKGROUND}

Umbilical cord is a connecting link between the foetus and the placenta through which foetal blood flows to and from the placenta. It extends from the foetal umbilicus to the foetal surface of the placenta ${ }^{1}$. It carries nutrients and oxygen to the baby and removes the baby's waste products. Umbilical cord is an extension of foetal cardiovascular system; its constituents are outer layer of amnion, inner porous Wharton jelly, two uterine arteries and one vein. ${ }^{2}$ Most umbilical cords are $40-70 \mathrm{~cm}$ long and few measures $<30 \mathrm{~cm}$ or $>100 \mathrm{~cm}$ long. Excessive long cords are linked with cord entanglements. ${ }^{3}$

Cord loops are frequently encountered and are caused by coiling around various foetal parts during foetal movements. ${ }^{3}$ Nuchal cord occurs when umbilical cord becomes wrapped around foetal neck 360 degree. The biometrics pattern of uterine, foetal, umbilical cord length coupled with placental and amniotic fluid volume could possibly be playing role in formation of nuchal cord. ${ }^{2}$ Single loop is reported in $20-34 \%$ cases, two loops in $2.5-5 \%$ and three loops in $0.2-0.5 \%{ }^{3}$ Ordinarily nuchal cord does not harm but occasionally they may be so tight that constriction of umbilical vessels and consequent hypoxia results especially during labour. ${ }^{2}$

Two types of nuchal cords have been identified. Type A A free sliding pattern which can undo itself. Type B - Nuchal loop that encircle the neck in a locked pattern and cannot undo itself. On delivery if cord is (1) Loose: it can easily be slipped over (2) Tight: slip over shoulder and deliver (3) Too tight: (a) Use somersault manoeuvre (b) clamp and cut the cord. During labour up to $20 \%$ foetuses with nuchal cord have moderate to severe variable heart rate decelerations and these are associated with a lower umbilical pH. Nuchal cord can be diagnosed antenatally by ultrasonography when combined with colour doppler imaging. ${ }^{2}$

The universal aim of maternity care provision is birth of a healthy baby to a healthy mother. Despite best efforts, occurrence of birth asphyxia continues to happen in obstetric practice and cord compression has been one of the postulated factors. About $20-60 \%$ of all foetal deaths are attributed to foetal asphyxia. Nuchal cord accident comprises 5-18\% of all foetal asphyxia cases. ${ }^{4}$ Such cases can have profound implications not only for the child and the parents but also for the practicing obstetrician. To avoid these untoward incidents in patients diagnosed with nuchal cord, clinicians adopt defensive practice and go for elective caesarean section straight way. These fears are believed to have contributed to the rising rate of primary $\mathrm{C}$ section nowadays. ${ }^{5}$

The incidence of elective and emergency $\mathrm{C}$ section is on the increase. In certain countries, figures beyond $30 \%$ are the norm. Over the period of time, $\mathrm{C}$ section has become a safer procedure, but it is a well-known fact that no surgical intervention is uneventful; Haemorrhage, anaesthesia related problems and infections are common complications. Moreover, the incidence of placenta accreta is on the increase with the increasing $\mathrm{C}$ section rate which is a worrisome factor. ${ }^{5}$

Mothers undergo enormous anxiety when they are told that their foetuses have nuchal cord as they simulate it to hanging noose and fear that their baby would be strangulated. It continues to challenge obstetricians as well and remains source of stress till patient is delivered. This study is an attempt made to see into that how much actual threat nuchal cord poses to mother as well as foetus and to identify various factors which are to be considered for safer delivery in these patients.

\section{METHODS}

This study was conducted in Sub-district hospital Akhnoor, Department of health and medical education Jammu. It was a prospective comparative study. Cases included in the study were with gestational age (37-41 weeks) admitted in labor room for delivery. The informed consent was taken from all the patients enrolled in the study. As no intervention has been done on the patients, a waiver was obtained for IEC approval.

\section{Inclusion Criteria \\ 1. Singleton pregnancy. \\ 2. Cephalic presentation. \\ 3. Term pregnancy. \\ 4. Live pregnancy.}

\section{Exclusion Criteria}

1. Pre-term labor.

2. Premature rupture of membranes.

3. Anomalous foetus.

4. Antenatal complications like preeclampsia, eclampsia, diabetes mellitus, severe anemia, cardiac disorders.

\section{Sample Size}

A minimum sample size of 54 patients in each group is calculated considering type- 1 error $\alpha=0.05$ and type- 2 error $\beta=0.2$ on the basis of previous study ${ }^{4}$ by using power and sample software (PS version 3.1.6). However, for better validation of results we have considered 100 patients in test group in which nuchal cord was identified during delivery, delivered either vaginally or by LSCS. These 100 cases were compared with 100 patients delivered without nuchal cord and were taken as control. For making study unbiased, every next patient of case was taken as control for finding out 100 case of nuchal cord, total number of patients observed were 462 Incidence of nuchal cord in our study came out $21.64 \%$. Patients with nuchal cord were separately categorized into (1) Single loop (2) Double loop (3) Triple loop (4) Quadruple loop.

Patients profile was recorded in prepared proforma in which detailed history and examination was recorded regarding age, parity, gestational age, labor spontaneous or induced, duration of labor, indication of LSCS, length of cord, number of turns of cord, outcome of baby in terms of gender, birth weight, Apgar score. Prior ultrasound detection of cord was noted. Foetal monitoring was done by intermittent auscultation with emphasis on rate, rhythm and tone of foetal heart especially before and after uterine contractions. If any abnormality recorded in FHR, it was noted. Status of liquor was checked till delivery. 


\section{Statistical Analysis}

All the collected data was entered in the Microsoft excel sheet and then analysed using computer software Microsoft excel SPSS version 22 for window. The qualitative data and quantitative data were reported as proportions and mean (sd) respectively. $\chi^{2}$-test was used for the comparison of qualitative variables and unpaired student t-test was used to test the significance between quantitative variables. A $p$-value less than or equal to 0.05 was considered as significant. All pvalues were two tails.

\section{RESULTS}

In our study most of the patients belonged to age group of 21 -25 years. Mean age of the test group is 25.56 years whereas control is 25.48 years. Difference is not statistically significant (p-0.88). It shows that there is no correlation of nuchal cord with any specific age group. Single loop was observed in 84 cases $(18.18 \%)$, double loop in 15 cases $(3.24 \%)$ and triple loop in 01 case $(0.21 \%)$. In none of the case quadruple or higher number of loops were observed. As the number of turns increased, incidence fell progressively. But as the number of loops increased, length of cord also increased. Mean length of single loop cord was $65.15 \mathrm{~cm}$ which increased to $76 \mathrm{~cm}$ in double loop and further increase was seen in triple loop which was $90 \mathrm{~cm}$. 17 cases and 2 controls had cord length more than $80 \mathrm{~cm}$. Difference is statistically significant $(\mathrm{p}<0.001)$ (Table 1) signifying excessive longer cords in nuchal cord group. Mean length of nuchal cord group in $\mathrm{cm}$ is 67.03 (12.41) and that of control is 60.12 (11.48). Difference is statistically significant with $\mathrm{p}<0.001$. Only 36 nuchal cords were pre detected by USG. Detection rate of double loop (37.5\%) was slightly higher than single loop (36.14\%) (Fig. 1).

In our study, maximum number of cases were primipara. As the parity increased, number of cases decreased in both groups. Mean gestational age of nuchal cord group in days is 275.95 (5.64) and control is 275.09 (6.36), difference was not found statistically significant $(\mathrm{p}=0.30)$.

When we compared duration of labour, majority patients had labour of 5-10 hrs. In both groups but in controls a greater number of patients had labour $<5 \mathrm{hrs}$. Which can be attributed to more multipara patients in control group as labour duration is less in multiparas.

Max number of patients had normal vaginal delivery in both groups, but more inductions were there in test group. Rate of LSCS was also higher in test group and difference was statistically significant (P-0.028) (Table 2). Most common indications of LSCS were AFD and failure of induction.

As far as baby's outcome was concerned, difference in newborn sex and birth weight was not statistically significant $(\mathrm{p}=0.23)$, birth weight in $\mathrm{kg}$ of test group is $3.04(0.43)$ and control is 2.96 (0.47) implying no correlation of nuchal cord with particular birth weight and sex.

Variable decelerations were seen only in test group (06 cases) signifying their strong association with nuchal cord. Meconium stained liquor was observed in 19 cases compared to only 8 in control group and difference was statistically significant (p- 0.023) (Table 3).

Although overall $\mathrm{A} / \mathrm{S}$ score was more in control group (Table 4) but low A/S $(<7)$ was seen in four patients in test group and one patient in controls. Difference was not statistically significant (p- 0.18) (Table 3). Only one baby was admitted in NICU with meconium aspiration syndrome delivered by LSCS.

\begin{tabular}{|ccccc|}
\hline Length of Cord & Test & Control & $\chi^{2}$ & $\mathbf{P}$ \\
$\leq 40 \mathrm{cms}$ & 2 & 05 & 1.326 & $0.249(\mathrm{NS})$ \\
$41-60 \mathrm{cms}$ & 33 & 44 & 2.542 & $0.11(\mathrm{NS})$ \\
$61-80 \mathrm{cms}$ & 48 & 49 & 0.02 & $0.88(\mathrm{NS})$ \\
$\geq 80 \mathrm{cms}$ & 17 & 02 & 13.02 & $<0.001(\mathrm{~S})$ \\
\hline Table 1. Comparison of Length of Cord in cms Using Chi Square \\
\hline
\end{tabular}

\begin{tabular}{|ccccc|}
\hline Mode of Delivery & Test & Control & $\chi^{2}$ & P \\
Spontaneous onset of Labour & 47 & 64 & 5.82 & $0.0158(\mathrm{~S})$ \\
Induced Labour & 24 & 20 & 0.464 & $0.496(\mathrm{NS})$ \\
LSCS & 29 & 16 & 4.82 & $0.028(\mathrm{~S})$ \\
\hline Table 2. Comparison of Mode of Onset of Labour Using Chi Square \\
\hline
\end{tabular}

\begin{tabular}{|ccccc|}
\hline Parameters & Test & Control & $\chi^{\mathbf{2}}$ & $\mathbf{P}$ \\
Meconium & 19 & 8 & 5.15 & $0.023(\mathrm{~S})$ \\
Decelerations & 3 & 1 & 1.015 & $0.3136(\mathrm{NS})$ \\
Low Apgar Score $(<7)$ & 4 & 1 & 1.837 & $0.18(\mathrm{NS})$ \\
\hline Table 3. Comparison of Foetal Distress & Using Chi Square \\
\hline
\end{tabular}

\begin{tabular}{|c|c|c|c|c|}
\hline Groups & $\mathbf{N}$ & $\overline{\mathbf{x}}$ & $\mathbf{T}$ & $\mathbf{P}$ \\
\hline Test & 100 & $9.05(1.54)$ & \multirow{2}{*}{-2.24} & \multirow{2}{*}{$0.02(\mathrm{~S})$} \\
\hline Control & 100 & $9.46(0.978)$ & & \\
\hline \multicolumn{5}{|c|}{ Table 4. Comparison of Apgar Score } \\
\hline
\end{tabular}

\begin{tabular}{|r|r|}
\hline 90 & \\
\hline 80 & \\
70 & \\
60 & \\
50 & \\
40 & \\
30 & \\
20 & \\
10 & \\
0 & \\
\hline & Single Loop Double Loop Triple Loop \\
\hline & No of cases $\quad$ Cases detected by USG \\
\hline
\end{tabular}

\section{DISCUSSION}

Majority patients in our study belonged to age group 21-25 years in both groups. This finding is similar to other related studies. This is because most of the patients reproduce in this age group only especially in rural India. However, Clapp et $\mathrm{al}^{2}$ reported increased incidence of nuchal cords with advancing gestation age.

Nuchal cord is a common feature observed during deliveries. We observed incidence of $21.64 \%$, which indicates that every $4^{\text {th }}$ to $5^{\text {th }}$ case is delivered with nuchal cord. Sheiner E, et al ${ }^{6}$ observed $14.7 \%$ incidence whereas incidence reported by Shrestha $\mathrm{NS}^{7}$ et al is $22.85 \%$. Henry et al ${ }^{8}$ observed incidence of tight nuchal cord $6.6 \%$ and $21.6 \%$ loose nuchal cord. We observed only three tight nuchal cord and all three were double loop. Mean length of cord was found more in test group ( $p<0.001)$. It implies chances of formation of nuchal loops are more in long cords. And as the length increases, number of loops also increase, and a linear correlation is seen. 
Various studies have been done on USG detection of nuchal cord prior to labour induction. Sensitivity and specificity of USG in detection of nuchal cord is low. Pregrine $\mathrm{E}$, et $\mathrm{al}^{9}$ reported $37.5 \%$ sensitivity of USG in diagnosing nuchal cord. In our study only 36 cases were predicted by USG in third trimester scan. Jouniaux et al showed USG to be gold standard when combined with colour Doppler imaging. This seems to correctly identify $72 \%$ of single and $94 \%$ of multiple nuchal cords with greatest sensitivity after 36 weeks. Sonologists can look for a divot sign on high resolution USG, a circular indentation of the foetal nuchal skin but care should be exercised not to confuse these findings with posterior cystic masses, fold of skin or amniotic fluid pockets. $^{2}$

Krakowiak defines short cord $<40 \mathrm{~cm}$ and occurs approximately in $6 \%$ of pregnancies. When short cord is around the neck, it is more likely to wrap tightly and are more often associated with decrease foetal movement, risk of cord compression, constriction and rupture. ${ }^{2}$ We found only two patients having cord length $<40 \mathrm{~cm}$. Naye et $\mathrm{al}^{2}$ observed that long cords $>70 \mathrm{~cm}$ have higher foetal entanglements. Findings are similar to our study. We found as the number of nuchal loops increased length of cord also increased. Mean length of single loop nuchal cord was $65.15 \mathrm{~cm}$ which increased to $76 \mathrm{~cm}$ in double loop and further increase was seen in triple loop which was $90 \mathrm{~cm}$.

Miser et al ${ }^{10}$ showed no influence of maternal age, race, parity on the incidence of nuchal cords. Our majority patients were primipara and para 1 . Although we had more females in study outcome, but difference was not statistically significant. Hatice et $\mathrm{al}^{11}$ showed male dominance $\mathrm{p}=0.014$. Miser et al also reported similar findings with incidence more common in male infants.

We had more inductions in test group, findings are similar to Sheiner E et al who also observed higher rate of labour inductions. $\mathrm{C}$ section rate was higher in our study but Shrestha et al observed higher rate of $\mathrm{C}$ section in the group without nuchal cord $(\mathrm{p}=0.029)$. Majority of our patients were operated for AFD (Meconium stained Liquor) and failure of induction. Hatice et al also observed AFD as leading cause of $\mathrm{C}$ section. One of the probable reasons for increased rate of LSCS was lower threshold for LSCS in pre-diagnosed cords and multiple loops

Schaffer et $\mathrm{al}^{2}$ and Miser et al observed low birth weight in nuchal cord group, but we didn't find any significant difference in birth weight. Many of infants with nuchal cord pass meconium which is probably an indication of vagal collapse. More babies passed meconium in our study in nuchal cord group and difference was statistically significant ( $\mathrm{p}=0.023)$. Our mean Apgar score was less in test group. Although low Apgar score $<7$ was more in nuchal cord group but difference was not statistically significant $p=0.18$. Shrestha et al had complications like foetal heart rate irregularities and meconium stain liquor were increased in nuchal cord group but statistically not significant $\mathrm{p}=0.108$. They also observed that Apgar score $<7$ at 1 low ( $p=0.010)$. Apgar score at 5 minutes and admission to NICU was not common. Haitice et al also didn't observe statistically significant difference in Apgar score of two groups.

\section{CONCLUSIONS}

Nuchal cord is a common entity observed almost in $20-25 \%$ of patients during delivery. Reasons for nuchal cord to arise are not clear but longer cords are associated with formation of nuchal cord. Locked type of umbilical cord is dangerous but can be diagnosed only after delivery of foetus. Diagnosis of umbilical cord is possible with USG and colour Doppler imaging. We need skilled sonologists to differentiate between loose, tight nuchal cord and should also confirm the number of loops in their findings. Incidence of tight cord is less and is more when associated with multiple loops. Mothers need reassurance and proper education to reduce anxiety when diagnosed with nuchal cord. During antenatal period, the patient should be monitored with DFMC, NST weekly or Doppler. During labour, close foetal heart monitoring is required in every case and LSCS should be performed only if signs of distress appear. Elective C section on USG diagnosed nuchal cord is a wrong practice and patient should be assessed holistically considering other parameters like age, parity, Bishop score and signs of foetal distress. Apgar score is not a sensitive indicator of acid base changes in nuchal cord patients. Umbilical artery must be sampled to detect hypercapnia.

Nuchal cord is a potential cause for perinatal distress and rarely significant long-term neurodevelopmental consequences occur in the developing foetus.

\section{REFERENCES}

[1] DC Dutta. Text book of Obstetrics. $6^{\text {th }}$ edn. Calcutta: New Central Book Agency (P) Ltd., 2004: p. 39.

[2] Peesay M. Nuchal cord \& its implications. Maternal Health, Neonatology \& Perinatology 2017;3(1):28.

[3] Cunningham FG, Leveno KJ, Bloom SL, et al. Williams Obstetrics. 25th edn. McGraw-Hill Education/ Medical 2018: p. 117.

[4] Khushboo J, Ruchi S, Madhu B, et al. Incidence of cord around neck \& its effects on labor \& neonatal outcome. Advances in Human Biology 2017;7(1):15-8.

[5] Bhide A, Arulkumaran S, Daftary SN, et al. Arias Practical Guide to high risk pregnancy \& delivery: a South Asian perspective. 4th edn. Elsevier India Pvt. Ltd., 2015: p. $359,385$.

[6] Sheiner E, Abramowicz JS, Levy A, et al. Nuchal cord is not associated with adverse perinatal outcome. Arch Gynecol Obst 2006;274(2):81-3.

[7] Shrestha NS, Singh N. Nuchal cord and perinatal outcome. Kathmandu University Medical Journal (KUMJ) 2007;5(3):360-3.

[8] Henry E, Andres RL, Christensen RD. Neonatal outcome following a tight nuchal cord. Journal of Perinatology 2013;33(3):231-4.

[9] Peregrine E, O'Beien P, Jauniaux E. Ultrasound detection of nuchal cord prior to labor induction \& the risk of caesarean section. Ultrasound Obst Gyneol 2005;25(2):160-4. 
[10] Miser WF. Weight outcome of infants born with nuchal cords. J Fam Pract 1992;34(4):441-5.
[11] Haitice A, Buke B, Peckcan MK, et al. Nuchal cord: Is it really the silent risk of pregnancy? The Journal of Maternal - Fetal \& Neonatal Medicine 2017;30(14):1730-3. 